\title{
Vivências de Psicólogos como Integrantes de Equipes Multidisciplinares em Hospital
}

\section{Psychologists' Lived Experiences as Members in Multidisciplinary Teams in Hospital}

\section{Vivencias de los Psicólogos como Miembros de Equipos Multidisciplinarios en Hospital}

\author{
Thaís de Castro Gazotti* \\ Universidade Paulista - UNIP, Limeira, São Paulo, Brasil
}

\section{Vera Engler Cury**}

Pontifícia Universidade Católica de Campinas - PUC-Campinas, Campinas, São Paulo, Brasil

\begin{abstract}
RESUMO
Objetivou-se analisar fenomenologicamente a experiência de psicólogos que atuam em equipes multidisciplinares em hospitais. Participaram do estudo nove psicólogos, sendo oito mulheres e um homem, de hospitais no estado de São Paulo. Realizaram-se encontros dialógicos individuais com os participantes registrados sob a forma de narrativas compreensivas, que visam descrever os principais elementos da experiência de cada participante. Em seguida, redigiu-se uma narrativa síntese contendo os elementos significativos das experiências para apreender a estrutura do fenômeno em foco. Concluiu-se que: psicólogos necessitam romper barreiras e sobrecarregam-se com demandas, pois as equipes dificilmente reconhecem suas funções e sua importância; batalham pelo princípio da integralidade junto à equipe; compreensão empática, aceitação positiva incondicional e confiança caracterizam suas atuações com pacientes, familiares e profissionais para um bom funcionamento da equipe; necessitam se cuidar para exercerem suas funções; boa formação em Psicologia e pós-graduação em Psicologia Hospitalar precedem atuação competente em equipe.

Palavras-chave: psicologia hospitalar, interdisciplinaridade, fenomenologia.
\end{abstract}

\section{ABSTRACT}

The aim was to analyze phenomenologically the experience of psychologists who work in multidisciplinary teams in hospitals. Nine psychologists participated in the study, being eight women and one man, from hospitals in the state of São Paulo. Individual dialogical meetings were held with registered participants in the form of comprehensive narratives, aimed at describing the main elements of each participant's experience. Then, a synthesis narrative was written containing the significant elements of the experiences to learn the structure of the phenomenon in focus. It was concluded that: psychologists need to break barriers and overload themselves with demands as the teams hardly recognize their functions and 
their importance; they fight for the principle of integrality with the team; empathic understanding, unconditional positive acceptance and trust characterize their actions with patients, family members and professionals for a good functioning of the team; they need to take care of themselves to exercise their functions; good training in Psychology and post-graduation in Hospital Psychology precede competent performance in the team.

Keywords: hospital psychology, interdisciplinarity, phenomenology.

\section{RESUMEN}

El objetivo era analizar fenomenológicamente la experiencia de psicólogos que trabajan en equipos multidisciplinares en hospitales. Nueve psicólogos participaron en el estudio, ocho mujeres y un hombre, de hospitales del estado de São Paulo. Se celebraron encuentros dialógicos individuales con los participantes inscritos en forma de narrativas comprensivas, con el fin de describir los principales elementos de la experiencia de cada uno de ellos. Luego, se escribió una narrativa de síntesis que contenía los elementos significativos de las experiencias para aprender la estructura del fenómeno en cuestión. Se concluyó que: los psicólogos necesitan romper barreras y están sobrecargados de demandas porque los equipos difícilmente reconocen sus funciones y su importancia; luchan por el principio de integralidad con el equipo; la comprensión empática, la aceptación positiva incondicional y la confianza caracterizan sus acciones con los pacientes, familiares y profesionales para el buen funcionamiento del equipo; necesitan cuidarse a sí mismos para ejercer sus funciones; la buena formación en Psicología y el posgrado en Psicología Hospitalaria preceden al desempeño competente en el equipo.

Palabras clave: psicología del hospital, interdisciplinariedad, fenomenología.

\section{O Psicólogo Hospitalar e a Equipe Multidisciplinar}

No Brasil, a atenção à saúde esteve centralizada no contexto hospitalar desde a década de 1940, em decorrência da implantação de uma proposta baseada no modelo clínico-assistencialista de cuidado. A partir da Constituição Federal de 1988, a saúde passou a ser compreendida como um direito de todos e um dever do Estado. Iniciou-se, então, a construção das diretrizes norteadoras da política pública denominada Sistema Único de Saúde (SUS), o qual teve sua modulação na década de 1990 (Leis no 8.080/1990 e $n^{\circ}$ $8.142 / 1990)$. A proposta do SUS está diretamente relacionada à $8^{a}$ Conferência Nacional de Saúde (CNS), realizada em 1986, que demarcou historicamente a reformulação do conceito de saúde no Brasil ao compreendê-la a partir do modelo biopsicossocial, que a concebe como uma conjugação de fatores físicos, psíquicos e sociais. A mudança no conceito de saúde promoveu novas formas de atuação da Psicologia na área da saúde; este campo de atuação é denominado, em outros países, Psicologia da Saúde - Health Psychology - caracterizando as práticas realizadas em instituições de nível primário, secundário e terciário; o conceito de "hospital" 
corresponde aos níveis secundário e terciário, apenas. Assim, a Psicologia Hospitalar pode ser compreendida como uma parte da Psicologia da Saúde, a qual é considerada um subcampo da Psicologia. No contexto hospitalar, o psicólogo não irá atuar diretamente nos processos de doença que cabem ao médico, mas irá auxiliar o paciente na busca pela reorganização do equilíbrio psicológico perdido em razão da doença (Melo, 2015).

Ao psicólogo especialista em Psicologia Hospitalar cabe a descrição apresentada na Resolução no 013/2007 do Conselho Federal de Psicologia, a qual consolida as resoluções relativas aos títulos de especialista em psicologia e dispõe sobre as normas e procedimentos para seu registro, como: atuação em instituições de saúde de nível secundário ou terciário, nas diferentes áreas da instituição (Ambulatório, Unidade de Tratamento Intensivo e Pronto Atendimento), em instituições de ensino superior e centros de pesquisa; assim como nas modalidades diversificadas de atendimento (psicoterapia individual, psicoterapia de grupo, grupos de profilaxia, consultoria e interconsulta). Sua atuação estabelece diferentes relações: médico-paciente, paciente-familiares, paciente-paciente, paciente-processo de adoecimento, hospitalização, repercussões emocionais e as questões subjetivas presentes entre os membros da equipe de saúde.

Neste campo de atuação busca-se promover e/ou recuperar a saúde física e mental daquele que recebe a assistência. Em relação ao trabalho com a equipe multidisciplinar, preferencialmente interdisciplinar, de acordo com a resolução acima citada, cabe ao psicólogo participar de decisões em relação à conduta a ser adotada pela equipe, objetivando promover apoio e segurança ao paciente e a seus familiares por meio de informações pertinentes à área de atuação, assim como impulsionar o suporte e o manejo voltado para possíveis dificuldades operacionais e/ou subjetivas dos membros da equipe.

Desenvolveu-se o conceito e a prática da interdisciplinaridade sob influência do princípio de integralidade proposto pelo SUS, pois a atenção à saúde passou a envolver práticas que levam em conta as complexidades presentes nos problemas de saúde (Chiararia, 2015). Deste modo, contrapõe-se ao paradigma cartesiano que se caracteriza pela fragmentação dos conhecimentos e pela criação das diversas especialidades.

Então, criaram-se as equipes multidisciplinares - também denominadas multiprofissionais -, as quais correspondem a grupos que atuam nas formações de redes em suas práticas diárias. Com o advento das equipes multidisciplinares, aumentou a inserção dos profissionais que atuam na área da saúde.

Assim, para que o psicólogo exerça seu trabalho no hospital, é fundamental que os demais profissionais compreendam o seu papel 
para um trabalho eficaz. No entanto, o desconhecimento das especialidades a respeito da atuação da Psicologia, não se restringe apenas ao contexto hospitalar, mas à ciência da Psicologia como um todo, havendo equívocos sobre o papel do psicólogo hospitalar e as demandas que lhe cabem, conforme apresentado no estudo de Kirchner, Granzotto e Menegatti (2012). Isto por vezes diminui o impacto da presença do psicólogo, levando-o a atuar em consonância ao que os demais membros da equipe esperam dele (Moré, Crepaldi, Queiroz, Wendt, \& Cardoso, 2004 citado por Morais, Castro, \& Souza, 2012).

Segundo Chiararia (2015), ao psicólogo em equipe multidisciplinar cabe atuar junto ao coletivo, às práticas preventivas e de tratamento, tendo em vista diagnosticar e compreender o conteúdo envolvido nas queixas, sintomas e patologias, assim como possibilitar estabelecer 0 vínculo entre o paciente e a equipe multiprofissional, e contribuir para a humanização no ambiente hospitalar. As funções do psicólogo no contexto hospitalar e como membro da equipe multidisciplinar, devem ter em vista a grande variedade de atividades designadas ao psicólogo hospitalar. No entanto, há obstáculos que precisam ser contornados para delinear melhor o espaço de atuação do psicólogo junto à equipe multidisciplinar, dentre eles destacam-se a relação e a comunicação entre a Psicologia e os demais saberes profissionais da equipe (Gazotti \& Prebianchi, 2014), que são base para o relacionamento interpessoal, o elemento fundamental para o bom funcionamento multidisciplinar.

\section{Delineamento da Pesquisa}

Este artigo decorre de uma pesquisa de Mestrado que objetivou descrever e compreender fenomenologicamente a experiência de psicólogos em relação às suas participações como membros de equipes multidisciplinares em contexto hospitalar. Para ser possível compreender a experiência dos participantes, adotou-se um delineamento qualitativo inspirado no método fenomenológico, desenvolvido pelo matemático e filósofo alemão Edmund Husserl (1859-1938).

Assim, o objeto de estudo é o próprio fenômeno em foco, o qual contempla as vivências humanas cotidianas, que são simbolizadas nos processos de imaginação e percepção, e que vão influir sobre as relações interpessoais. O olhar fenomenológico volta-se para a subjetividade de modo a evidenciar a essência de cada aspecto que a constitui (Husserl, 2002).

O método fenomenológico aplicado às pesquisas na área da Psicologia, caracteriza-se por princípios inter-relacionados, são eles: a redução fenomenológica (suspensão dos conhecimentos prévios e 
teóricos sobre o fenômeno em estudo, de modo que os conhecimentos não são negados em sua veracidade, apenas são colocados de lado no momento de conhecer o fenômeno); a descrição da experiência do participante (apresentar as estruturas essenciais do fenômeno) (Giorgi, 1997 citado por Finlay, 2009); e a redução eidética ou transcendental (busca pelas essências, as quais correspondem à estrutura imutável do fenômeno que possibilita àquele que o percebe intuir seus sentidos) (Husserl, 2002).

$\mathrm{Na}$ pesquisa fenomenológica o pesquisador assume papel ativo importante ao abrir-se à experiência do outro a partir de uma atitude fenomenológica (Finlay, 2009). Esta atitude aproxima-se daquelas descritas por Carl. R. Rogers (1902-1987), psicólogo norte-americano que desenvolveu a Abordagem Centrada na Pessoa, que são: aceitação positiva incondicional (cada pessoa deve ser valorizada positivamente de maneira incondicional), compreensão empática (atitude de abertura para o referencial interno do outro e seus significados), e autenticidade ou congruência (estar presente como pessoa íntegra e genuína consigo mesma e na relação com o outro) (Rogers \& Wood, 2008).

\section{Coleta e Análise de Dados}

A estratégia metodológica utilizada para coleta de dados foi a narrativa. Em um encontro dialógico entre pesquisador e participante, o relato do participante é compreendido como um narrar de sua história e de seus significados simbolizados. Ao pesquisador cabe escrever as narrativas em vista de descrever (a partir de sua consciência), compreender e analisar os significados que emergiram nos encontros com os participantes sobre suas experiências diante do fenômeno em foco.

O processo de análise de dados inicia-se pela construção de narrativas compreensivas referentes ao encontro dialógico com cada participante para aproximar-se das significações vividas durante cada encontro. Logo após, ocorreu a leitura de cada narrativa aos demais pesquisadores do Grupo de Pesquisa Institucional, objetivando receber contribuições para o processo de análise dos encontros. $\mathrm{Na}$ sequência, as narrativas compreensivas foram lidas em conjunto para dar origem à narrativa síntese, que contempla a compreensão sobre os elementos significativos das experiências de todos os participantes, em busca dos elementos estruturais do fenômeno humano em estudo.

\section{Participantes}

Após a aprovação da pesquisa pelo Comitê de Ética em Pesquisa com Seres Humanos (parecer no 1.365.235), a pesquisadora entrou em 
contato com alguns psicólogos e convidou-os a participarem do estudo. Nove psicólogos hospitalares, sendo oito mulheres e um homem, concordaram em participar. Foram, então, realizados encontros dialógicos únicos e individuais com cada participante, no início dos quais foi entregue e assinado o Termo de Consentimento Livre e Esclarecido. Na sequência, a pesquisadora apresentou a questão norteadora: "Gostaria que me contasse sobre a sua experiência, como psicólogo hospitalar, ao participar de uma equipe multidisciplinar no hospital". Os nomes dos participantes são fictícios em virtude de preservar o sigilo diante dos dados de identificação dos participantes.

\begin{tabular}{|c|c|c|c|c|}
\hline $\begin{array}{l}\text { Narrativas } \\
\text { Participantes }\end{array}$ & $\begin{array}{c}\text { Ano } \\
\text { Conclusão } \\
\text { Curso } \\
\text { Psicologia }\end{array}$ & $\begin{array}{c}\text { Formação em Pós- } \\
\text { Graduação }\end{array}$ & $\begin{array}{c}\text { Tempo de } \\
\text { Atuação } \\
\text { em } \\
\text { Hospital }\end{array}$ & $\begin{array}{l}\text { Tipo de } \\
\text { Hospital }\end{array}$ \\
\hline $\begin{array}{l}\text { Narrativa } 1 \\
\text { Andressa }\end{array}$ & 2010 & Especialização & 6 anos & Geral \\
\hline $\begin{array}{l}\text { Narrativa } 2 \\
\text { Thalita }\end{array}$ & 2008 & Aprimoramento & 4 anos & Cardiológico \\
\hline $\begin{array}{l}\text { Narrativa } 3 \\
\text { Alessandra }\end{array}$ & 2006 & Aprimoramento & 10 anos & $\begin{array}{c}\text { Geral } \\
\text { Universitário }\end{array}$ \\
\hline $\begin{array}{l}\text { Narrativa } 4 \\
\text { Gabriela }\end{array}$ & 2011 & $\begin{array}{l}\text { Residência } \\
\text { Multidisciplinar }\end{array}$ & $11 / 2$ anos & Geral \\
\hline $\begin{array}{l}\text { Narrativa } 5 \\
\text { Jéssica }\end{array}$ & 2013 & Especialização & $11 / 2$ anos & Cardiológico \\
\hline $\begin{array}{l}\text { Narrativa } 6 \\
\text { Rodrigo }\end{array}$ & 2012 & $\begin{array}{l}\text { Residência } \\
\text { Multidisciplinar }\end{array}$ & $11 / 2$ anos & $\begin{array}{c}\text { Reconstrução } \\
\text { Plástica }\end{array}$ \\
\hline $\begin{array}{l}\text { Narrativa } 7 \\
\text { Soraya }\end{array}$ & 2006 & $\begin{array}{l}\text { Aprimoramento/ } \\
\text { Especialização }\end{array}$ & 6 anos & Geral \\
\hline $\begin{array}{l}\text { Narrativa } 8 \\
\text { Isadora }\end{array}$ & 2011 & $\begin{array}{l}\text { Residência } \\
\text { Multidisciplinar }\end{array}$ & 2 anos & Cardiológico \\
\hline $\begin{array}{l}\text { Narrativa } 9 \\
\text { Alana }\end{array}$ & 2012 & $\begin{array}{c}\text { Residência } \\
\text { Multidisciplinar }\end{array}$ & $1 / 2$ ano & $\begin{array}{c}\text { Geral } \\
\text { Universitário }\end{array}$ \\
\hline
\end{tabular}

De acordo com a Tabela 1, a respeitos dos participantes, observa-se que: seis $(66,6 \%)$ participantes encontram-se na faixa etária de 26 a 30 anos, dois (22,2\%) encontram-se na faixa etária de 31 a 35 anos, e um $(11,1 \%)$ encontra-se na faixa etária de 41 a 45 anos. Dos estados brasileiros onde graduaram-se em Psicologia, cinco $(55,5 \%)$ participantes realizaram a graduação no estado de São Paulo, um $(11,1 \%)$ graduou-se em Brasília, um $(11,1 \%)$ graduou-se no Paraná, um $(11,1 \%)$ graduou-se em Minas Gerais e um $(11,1 \%)$ graduou-se na Bahia.

A seguir, objetivando exemplificar, apresenta-se um trecho de uma narrativa escrita sobre o encontro da pesquisadora com a psicóloga Thalita: 
"(...) Tais experiências a fizeram notar que o mais importante na relação com uma equipe é o tempo de conhecimento entre os profissionais, pois quando conhecem a psicóloga há um tempo é mais fácil considerarem suas avaliações. Ou seja, ao iniciar sua participação no grupo, o psicólogo tem que conquistar seu espaço e ganhar credibilidade. (...)."

\section{Os Resultados em Discussão}

Para uma boa atuação do psicólogo em contexto hospitalar, a transposição de modelos anteriores de atuação a este contexto é inadequada devido às exigências específicas que o caracterizam (Santos \& Jacó-Vilela, 2009 citado por Azevêdo \& Crepaldi, 2016). Em decorrência disto, pode-se observar nos dados da Tabela 1 que todos os participantes desta pesquisa cursaram algum tipo de Programa de Pós-Graduação Lato Sensu em Psicologia Hospitalar após a conclusão do curso de Graduação em Psicologia, priorizando os cursos disponíveis na época, devido às exigências específicas que caracterizam este campo de atuação.

A partir das narrativas escritas sobre os encontros, pôde-se descrever os elementos estruturantes das experiências dos participantes. Embora os elementos sejam apresentados distintamente, guardam relações entre si.

\section{A Necessidade de "Cavar Espaço" para Conquistar Respeito e Reconhecimento por Parte da Equipe}

Este elemento mostrou-se como fundamental para que os psicólogos pudessem iniciar efetivamente suas atividades junto à equipe multidisciplinar no contexto hospitalar. O espaço que buscam conquistar diz respeito tanto a atender às demandas hospitalares que Ihes cabem, quanto à participação em equipe multidisciplinar.

Os participantes priorizam construir uma relação junto à equipe em vista de criar, primordialmente, um vínculo de confiança entre os profissionais, enquanto pessoas e especialistas. A equipe segue um delineamento único para uma atuação em conjunto, por isso é importante que seja estabelecido um envolvimento enquanto grupo.

De acordo com Rogers (1976), um grupo pode se formar de diversas maneiras e com diversos propósitos, mas há uma qualidade que os aproximam: a possibilidade dos envolvidos vivenciarem uma experiência intensiva que influencia no desenvolvimento grupal e pessoal. A participação em grupo proporciona o desenvolvimento de um relacionamento que possibilita mudanças construtivas a partir de um processo de crescimento intersubjetivo. 
Este crescimento intersubjetivo pode ocasionar em mudanças: na pessoa que vivencia a aceitação positiva na equipe e reciprocamente, podendo mudar o próprio autoconceito e suas atitudes ao sentir-se num clima grupal empático; e no grupo, devido à convivência cotidiana levar ao compartilhamento de conhecimentos, procedimentos e modos de intervenção que desenvolve sentimentos de segurança, beneficiando a integralidade das ações. Com isso, é provável que ocorram mudanças na própria instituição a partir da maior consolidação e protagonismo das equipes.

O desejo de participar efetivamente da equipe e contribuir para a qualificação do cuidado aos pacientes, transcende as características elencadas pela Resolução no 013/2007 do CFP, que delimita os possíveis moldes de ações referentes a cada tipo de demanda que 0 psicólogo hospitalar é solicitado em relação: ao paciente, à equipe, aos familiares e às diversas sistematizações da atuação do psicólogo hospitalar encontradas na literatura (Nogueira-Martins, 2012; Gazotti \& Prebianchi, 2014).

De acordo com os participantes desta pesquisa, é essencial para uma boa atuação, que o psicólogo hospitalar estabeleça uma relação grupal coesa, pois, ao priorizar um conhecimento coletivo, os saberes de cada especialidade são compartilhados de modo a potencializar a qualidade da atuação interdisciplinar (Bezerra, 2016).

\section{A Sobrecarga de Demandas que Recaem sobre o Psicólogo ao Participar de uma Equipe Multidisciplinar}

Os participantes descreveram a existência de diversas barreiras a serem ultrapassadas para que possam participar junto às outras especialidades como um grupo, de modo a poderem atuar a seus próprios modos diante das demandas e das relações que estabelecem. Suas atuações em equipes multidisciplinares têm em vista oferecer atenção às demandas de modo integral.

Consideram os obstáculos: a falta de compreensão dos profissionais da equipe diante do processo subjetivo de adoecimento e a falta de suporte emocional para lidar com as demandas e exigências emocionais dos pacientes, por sentirem-se vulneráveis a vivenciar os mesmos sentimentos que os pacientes ao não disporem de recursos psicológicos para lidar com esta demanda. Um estudo realizado em Londres apresentou as tensões emocionais que os profissionais da saúde, no caso os enfermeiros, podem vivenciar no contexto hospitalar: observou-se 0 aumento de ansiedade, angústia e frustração em decorrência das situações vividas nas condições de trabalho, junto a sentimentos contraditórios em relação aos pacientes - piedade, compaixão, amor; culpa, ansiedade, ódio e ressentimento (Nogueira-Martins, 2012). 
Uma vez que o psicólogo hospitalar recebe uma solicitação de intervenção e constata que decorre da equipe ou de algum profissional individualmente, Ihe é exigido realizar um trabalho duplo: atender ao paciente para fazer sua própria avaliação sobre seu estado psicológico e emocional naquele momento, e então, retornar à equipe para analisar, agora, ambas as demandas - a do paciente e a da equipe - e buscar solucioná-las em conjunto. Assim, deve ser reconhecido que o trabalho do psicólogo hospitalar junto à equipe não se limita a fazer parte dela de modo a caracterizá-la enquanto multidisciplinar; mas também, oferecer suporte e auxílio psicológico para as dificuldades práticas e subjetivas dos profissionais diante da sua prática cotidiana.

Segundo Oliveira (2013), a prática cotidiana no contexto hospitalar representa um desafio aos profissionais da equipe por vivenciarem situações que não estão descritas nos livros e para as quais não foram preparados, tanto do ponto de vista teórico, quanto técnico. Cabe ao psicólogo contribuir para a avaliação desses aspectos em relação aos pacientes atendidos e estar aberto a acolher as reações emocionais dos próprios colegas de equipe.

\section{A Preocupação com a Integralidade do Cuidado Ofertado}

Para os participantes, no momento em que os membros da equipe compreendem o papel do psicólogo hospitalar e reconhecem a necessidade de dar importância aos aspectos subjetivos e emocionais dos pacientes, torna-se possível haver um trabalho com maior intercâmbio entre os diversos saberes. Anteriormente a isto, os profissionais irão agir isoladamente e à sua própria maneira com as demandas emocionais dos pacientes, assim, excedem suas próprias competências e vêm a ocupar o lugar destinado ao psicólogo hospitalar. Estes aspectos somente são ressaltados caso haja um psicólogo na equipe para trazê-los à tona e que possa orientar tais compreensões.

Estes obstáculos mostram a importância de haver conhecimento por parte dos membros da equipe a respeito dos conceitos sobre cuidado biopsicossocial, integralidade e interdisciplinaridade no contexto hospitalar. A presença do psicólogo contribui para concretizar práticas de natureza interdisciplinar do cuidado devido à sua atuação principalmente enquanto interconsultor (Gazotti \& Prebianchi, 2014), uma vez que, na maior parte das vezes, os aspectos emocionais e sociais influenciam as enfermidades trazendo consequências desorganizadoras para a vida cotidiana do paciente e de seus familiares. O psicólogo hospitalar propicia à equipe, com maior facilidade, um olhar amplo e inter-relacionado de todos os aspectos presentes no cuidado aos pacientes do ponto de vista relacional. 


\section{O Especialista no Cuidado Subjetivo e Emocional e a Atitude Empática}

Em situações onde as solicitações de atendimento psicológico decorrem de demandas da própria equipe, o psicólogo, enquanto especialista no cuidado aos aspectos subjetivos e emocionais, percebe a passagem de responsabilidade da equipe que busca se afastar das demandas que lhe causam angústia. A presença do psicólogo hospitalar torna-se essencial, pois percebe que, caso a equipe venha a enfrentar sozinha tais circunstâncias, pode prejudicar o equilíbrio psicológico e emocional das demais especialidades. Desse modo, o trabalho do psicólogo em mediar a relação equipe/profissional-paciente é intensificado. O distanciamento dos profissionais frente aos pacientes pode produzir a eles sofrimento, pois conduz a um sentimento de insegurança diante da doença, do prognóstico e do profissional (Riba \& Juver, 2009 citado por Barreto \& Castro, 2015).

Os participantes compreendem a atitude da equipe nestas circunstâncias, e enfatizam a intenção de desenvolver na equipe o olhar empático diante do processo de adoecimento e dos aspectos subjetivos presentes para que haja uma corresponsabilização do cuidado. Preocupam-se ainda, em atuar em relação aos pacientes e à equipe a partir de uma atitude empática.

De acordo com Rogers e Wood (2008), a atitude empática representa a possibilidade de "sentir o mundo privado do outro como se fosse o seu, mas sem perder a qualidade do 'como se'" (p. 151). Esta atitude possibilita ao outro - no caso, o psicólogo hospitalar - compreender aquilo que um - o paciente, a equipe ou um profissional - já conhece; assim como também capacita o psicólogo a entrar em contato com os significados da experiência do outro, expressa no momento em que este tem apenas breve ou nenhum conhecimento.

Isto proporcionará evoluir a qualidade dos relacionamentos, cultivar o olhar para a perspectiva do paciente de maneira ampliada e possibilitar aos profissionais compreenderem de maneira integrada, as demandas, os quadros clínicos, o modo subjetivo de cada paciente lidar com seu processo de adoecimento, e aos próprios profissionais poderem compreender seus sentimentos provenientes da relação de cuidado. Trata-se de uma oportunidade do psicólogo hospitalar de auxiliar os demais profissionais a desenvolverem a atitude de empatia diante dos pacientes para evoluírem os relacionamentos estabelecidos e, assim, estabelecerem devidamente suas atuações, tanto individualmente, quanto em grupo. 


\section{A Valorização do Psicólogo Hospitalar pela Equipe Desenvolve- se a partir de uma Relação de Confiança Mútua}

Para os participantes, somente a partir do momento em que suas atuações entram em cena e eles participam em conjunto das intervenções da equipe, podem iniciar um trabalho interdisciplinar com olhar amplo para as necessidades subjetivas e emocionais. Caso contrário, apenas Ihes caberia um papel protocolar em relação aos pacientes e aos procedimentos de avaliação desenvolvidos pela equipe.

Partindo da percepção dos participantes, a respeito da defasagem na compreensão dos demais profissionais sobre suas atuações, isto provoca um distanciamento entre os profissionais da saúde e os psicólogos porque, aos olhos da equipe multidisciplinar, o psicólogo não é tão fundamental quanto os demais no cuidado à saúde.

O fato da Psicologia ser parte das Ciências Humanas e das Ciências da Saúde, promove nos profissionais da saúde uma certa desconfiança sobre as afinidades possíveis para uma atuação conjunta. As demais especialidades, tais como Medicina, Enfermagem, Fisioterapia, Fonoaudiologia, entre outras, parecem se reconhecer reciprocamente em relação aos procedimentos e visões sobre o binômio saúde-doença. No caso da Psicologia, a perspectiva contempla aspectos emocionais nas relações subjetivas e intersubjetivas, cujo objeto difere das ciências tradicionalmente consideradas da área da saúde. Ela tem como objeto de estudo o funcionamento psicológico dos indivíduos, o qual não é visível, palpável, mensurável, qualificável e, tampouco, passível de qualificação dos resultados obtidos em um tratamento da mesma forma que é possível para as Ciências Médicas (Melo, 2015). Por isso, a literatura apresenta (Kirchner et al., 2012) conflitos gerados devido à busca, das demais áreas da saúde, por resultados concretos do tratamento realizado pelo psicólogo, sem compreenderem 0 funcionamento e o olhar desta ciência para o seu objeto de estudo.

Os participantes relataram que, a partir da construção de uma relação grupal apropriada com a equipe e através da participação nas reuniões semanais, puderam ter a oportunidade de estabelecer atividades que promovam o diálogo e a compreensão entre os diferentes pontos de vista de cada especialidade que possibilitam aos profissionais desenvolver, entre si, práticas mais cooperativas e centradas no paciente (Motta \& Pacheco, 2014).

No momento em que este nível de relação é alcançado, os participantes percebem que as atitudes para com eles, com as demandas ou com os cuidados psicológicos também mudam. Os demais profissionais passam a valorizar suas presenças e participações, expressando sua valorização ao solicitarem avaliações e suporte psicológico e ao considerarem suas contribuições, suas 
análises sobre os casos e suas propostas de manejo das relações com os pacientes, bem como com seus familiares em determinadas situações que exijam sensibilidade e acolhimento.

\section{Cuidar de Si Mesmo como Oportunidade de Crescimento Pessoal}

Para que seja possível estarem dispostos a auxiliar os demais em suas necessidades e estarem presentes e íntegros em todas as relações, os psicólogos precisam olhar para si mesmos, enquanto pessoas e profissionais, de modo a se prepararem para as novas situações e demandas que os desafiam. Pois, uma das funções do psicólogo é lidar com conflitos psicológicos que se desenvolvem nas interações entre profissionais, membros das equipes, pacientes e familiares que demandam uma escuta qualificada.

No caso de uma intermediação entre a equipe e o paciente, os participantes ressaltam a preocupação em se manterem o máximo possível em uma posição de neutralidade. Para tanto, é necessário que o psicólogo tenha claro para si esta característica peculiar de seu trabalho. Isto torna a sua preparação psicológica essencial para que atue de maneira segura e imparcial, pois também estão presentes questões particulares.

Os participantes também sentem necessidade de poder ter um momento para relaxar da tensão do trabalho. Por isso, ressaltam a importância de terem contato com colegas psicólogos próximos, no mesmo hospital, para discutirem mais a fundo os casos e poderem expressar suas angústias. Do contrário, o trabalho pode vir a se tornar muito solitário.

Os momentos vividos rememorados e as diferenças nas formas como os participantes os significam e os interpretam, possibilitaram perceber que houve um processo de crescimento pessoal e profissional em cada um que representa o processo de tendência à autoatualização. A tendência à autoatualização corresponde a um conjunto de forças organísmicas que movimentam o indivíduo em direção ao seu crescimento e que podem ser potencializadas quando sob condições físicas e psicológicas facilitadoras, saudáveis e favoráveis (Rogers \& Wood, 2008). De acordo com os mesmos autores, essa tendência, presente em todas as pessoas, direciona o organismo a entrar em contato com suas atitudes, sentimentos e emoções de modo a explorá-los, genuinamente, no seu próprio ritmo e com suas próprias simbolizações.

O crescimento psicológico favorece os participantes a terem maior flexibilidade pessoal e psicológica para lidar com as adversidades e obstáculos que, inevitavelmente, estão presentes no contexto em que atuam. Tornam-se mais munidos, psicológica e emocionalmente, de 
recursos internos para enfrentar tais barreiras e para agir de modo mais criativo.

\section{Considerações Finais}

A atuação do psicólogo em uma equipe multidisciplinar que reconhece a sua presença como agregando qualidade ao grupo, proporciona aos pacientes um atendimento integrado em função do compartilhamento de saberes entre membros da equipe. Os participantes foram bastante enfáticos sobre a importância da existência de uma relação bem construída e sedimentada em confiança mútua entre todos os membros da equipe multidisciplinar.

Este trabalho pôde descrever as vivências dos psicólogos e compreender a experiência da atuação do psicólogo inserido na equipe multidisciplinar em hospitais. O número de participantes não permite a generalização dos resultados, mas as experiências apresentadas possibilitam ao leitor considerar a relevância da discussão deste trabalho a partir da sua própria situação vivida.

As dificuldades presentes no trabalho realizado pelo psicólogo hospitalar são temas que precisam ser continuamente pesquisados para que se possa analisar novas propostas de atuação e explorar as atividades que podem ser exercidas em conjunto por uma equipe multidisciplinar. Tem-se em vista que cada vez mais seja possibilitada a atuação interdisciplinar e o cuidado centrado na pessoa integrados à prática das especialidades da área da saúde, principalmente quando atuando em equipe.

Esta forma diferenciada de olhar o fenômeno, a partir de uma relação intersubjetiva que a pesquisa apresenta, possibilitou apresentá-lo de modo a abranger diversas facetas que o constituem, privilegiando as vivências dos participantes. Novos estudos que enfatizem os elementos significativos das vivências de psicólogos serão bemvindos para aprimorar os diversos níveis de formação responsáveis pelo desenvolvimento de competências e habilidades para atuar na área da saúde.

\section{Referências}

Azevêdo, A. V. S., \& Crepaldi, M. A. (2016). A Psicologia no hospital geral: aspectos históricos, conceituais e práticos. Estudos de Psicologia (Campinas), 33(4), 573-585. doi:10.1590/198202752016000400002

Barreto, S. M., \& Castro, E. K. (2015). Critérios de médicos oncologistas para encaminhamento psicológico em cuidados 
paliativos. Psicologia: Ciência e Profissão, 35(1), 69-82. doi:10.1590/1982-3703000202013

Bezerra, M. C. S. (2016). A experiência de psicólogos como residentes em programa multiprofissional em saúde. Dissertação de Mestrado. Pontifícia Universidade Católica de Campinas, SP. Recuperado de http://tede.bibliotecadigital.puccampinas.edu.br:8080/jspui/handle/tede/905?mode=full

Chiararia, D. O. (2015). Inserção da psicologia no contexto hospitalar sob a ótica do matriciamento. Trabalho de Conclusão de Curso, Programa de Aprimoramento Profissional/SES-Fundap em Psicologia Hospitalar, Faculdade de Medicina de Marília, SP. Recuperado de http://ses.sp.bvs.br/lildbi/docsonline/get.php?id=5680

Finlay, L. (2009). Debating Phenomenological Research Methods. Phenomenology \& Practice, 3(1), 6-35. Recuperado de https://ejournals.library.ualberta.ca/index.php/pandpr/article/vi ew/19818/15336

Gazotti, T. C. \& Prebianchi, H. B. (2014). Caracterização da interconsulta psicológica em um hospital geral. Psicologia: Teoria e Prática, 16(1), 18-30. doi:10.15348/1980.6906/psicologia.v16n1p18-30

Husserl, E. (2002). A crise da humanidade europeia e a filosofia. Porto Alegre: EDIPUCRS.

Kirchner, L. F., Granzotto, M. D., \& Menegatti, C. L. (2012). Concepções da equipe de saúde de um hospital de Curitiba/Paraná sobre a prática de psicologia. Estudos Interdisciplinares em Psicologia (Londrina), 3(1), 24-40. doi:10.5433/2236-6407.2012v3n1p24

Melo, C. B. (2015). História da psicologia e a inserção do psicólogo no hospital. In L. C. Santos, E. M. F. Miranda \& E. L. Nogueira (Orgs.), Psicologia, saúde e hospital: Contribuições para a prática profissional (pp. 19-32). Belo Horizonte: Artesã.

Morais, J. L., Castro, E. S. A., \& Souza, A. M. (2012). A inserção do psicólogo na residência multiprofissional em saúde: Um relato de experiência em oncologia. Psicologia em Revista (Belo Horizonte), 18(3), 389-401. Recuperado de http://pepsic.bvsalud.org/pdf/per/v18n3/v18n3a04.pdf

Motta, L. B., \& Pacheco, L. C. (2014). Integranting medical and health multiprofissional residency programs: The experience in building an interprofessional curriculum for health profissionals in Brazil. Education for Health, 27(1), 83-88. doi: $10.4103 / 1357-6283.134331$

Nogueira-Martins, L. A. (2012). Saúde mental dos profissionais da saúde. In N. J. Botega (Org.), Prática psiquiátrica no hospital geral: Interconsulta e emergência (pp. 98-112). Porto Alegre: Artmed. 
Oliveira, A. E. G. (2013). A experiência de profissionais da saúde no cuidado a pacientes em ambulatório de quimioterapia. Dissertação de Mestrado. Pontifícia Universidade Católica de Campinas, SP. Recuperado de http://tede.bibliotecadigital.puccampinas.edu.br:8080/jspui/handle/tede/320

Rogers, C. R. (1976). Grupo de encontro. Lisboa: Moraes Editores.

Rogers, C. R., \& Wood, J. K. (2008). A abordagem centrada na pessoa. Vitória: EDUFES.

\section{Endereço para correspondência \\ Thaís de Castro Gazotti}

Rua Tiradentes, 235 apto 141, Centro, CEP 13480-080, Limeira - SP, Brasil

Endereço eletrônico: thaisgazotti@yahoo.com

\section{Vera Engler Cury}

Rua João Stanis, 05, Taquaral, CEP 13076-270, Campinas - SP, Brasil

Endereço eletrônico: vengler@puc-campinas.edu.br

Recebido em: 04/02/2019

Aceito em: 13/11/2019

\section{Notas}

* Doutoranda FMUSP. Mestre Psicologia PUC-Campinas. Formação Transtornos Alimentares (AMBULIM-IPq-HC-FMUSP). Psicóloga AMBULIM e clínica. Docente UNIP-Limeira.

** Especialista Psicologia Clínica. Mestre Psicologia Clínica IPUSP. Doutora Saúde Mental FCM UNICAMP. Docente titular Graduação e Pós-Graduação em Psicologia PUC-Campinas.

Financiamento: Bolsa CNPq.

Este artigo de revista Estudos e Pesquisas em Psicologia é licenciado sob uma Licença Creative Commons Atribuição-Não Comercial 3.0 Não Adaptada. 